Series A

I. MATHEMATICA

318

\title{
ZUR THEORIE \\ DER VEKTORWERTIGEN \\ DIFFERENTIALFORMEN
}

VON

H. HAAHTI und T. KLEMOLA

HEL S N K I 1962

S U O M A A I NEN TIEDEAKATEMIA

https://doi.org/10.5186/aasfm.1963.318 
Am 8 Dezember vorgelegt von F. Nevandinna und G. Järnefelt 


\section{§1. Einleitung}

Das Thema dieser Arbeit besteht in der Untersuchung einer Verallgemeinerung der vektoriellen totalen Differentialgleichung

$$
y^{\prime}(x)=A(x) y(x)+a(x)
$$

wo $y$ die unbekannte vektorielle Funktion ist. $\left.{ }^{1}\right)$ Anstatt der Funktion $y$ wird die Unbekannte eine vektorwertige Differentialform $f$ sein; die der Obigen entsprechende Gleichung ist dann

$$
(d f)(x)=A(x) \wedge f(x)+a(x),
$$

wo nun $d f$ den Rotor oder die äussere Ableitung von $f$ bedeutet und $a$ eine vorgegebene vektorwertige Differentialform ist. Das Produkt $\wedge$ ist eine natürliche Erweiterung des äusseren Produktes.

Die in dieser Schrift rorkommenden Bezeichnungen und Begriffe stammen zum grossen Teil aus der Monographie [3] von F. und R. Nevandinna, wo auch die Grundzüge der Theorie der Gleichung (1.1) (eigentlich nur des homogenen Falles $a=0$ ) angegeben sind ([3], IV.2).

Im zweitem Paragraphen dieser Arbeit werden die verwendeten Begriffe und die Terminologie eingeführt. Es folgt dann eine genauere Problemstellung (§3) und Diskussion des homogenen Falles (\$4). In $\S 5$ wird hiernach die inhomogene Gleichung (1.2) mit Hilfe der in den vorangegangenen Paragraphen ausgearbeiteten Methoden behandelt. Im letzten Paragraphen beschäftigen wir uns mit zwei differentiellen Folgen ( (6.2) und (6.6)), zu welchen die Gleichung (1.2) führt.

Als Definitionsbereich der Lösungen benutzen wir eine geschlossene Kugel $U$ in einem endlich-dimensionalen normierten Raum, so dass alle Problemstellungen in dieser Arbeit lokal oder semi-lokal sind.

1) Hier variiert $x$ bzw. $y$ in einem endlich-dimensionalen reellen Vektorraum $R_{x}$ bzw. $R_{y} ; a$ ist eine Funktion von $x$, deren Werte lineare Abbildungen von $R_{x}$ nach $R_{y}$ sind, und $A(x)$ ist für jedes $x$ eine bilineare Abbildung von $R_{x} \times R_{y}$ nach $R_{y}$. 


\section{§ 2. Vorbereitende Betrachtungen}

2.1. Sind $X$ und $Y$ zwei lineare reelle Räume, so bezeichnen wir mit Hom $(X, Y)$ die Menge der linearen Abbildungen von $X$ in $Y$. Es seien jetzt $R_{x}$ und $R_{y}$ zwei vorgegebene lineare normierte reelle Räume endlicher Dimension (mit $\operatorname{dim} R_{x}=m$, $\operatorname{dim} R_{y}=n$ ). Wir setzen dann

$$
V_{p}=\operatorname{Hom}\left(\wedge^{p} R_{x} ; R_{y}\right) ; W_{p}=\operatorname{Hom}\left(\wedge^{p} R_{x} ; \operatorname{Hom}\left(R_{y} ; R_{y}\right)\right) .
$$

Hier ist $\wedge^{p} R_{x}$ die p-te äussere Potenz von $R_{x}$. Für $p=0$ haben wir (nach geeigneten Identifikationen)

$$
V_{0}=R_{y} ; \quad W_{0}=\operatorname{Hom}\left(R_{y} ; R_{y}\right) .
$$

Der Raum Hom $\left(\wedge^{p} R_{x} ; R\right)$ der reellwertigen $p$-linearen und alternierenden Funktionen kann als ein linearer Unterraum in $W_{p}$ aufgefasst werden, indem man einer reellwertigen Funktion $\alpha_{p}$ das $W_{p}$-Element $A_{p}=\alpha \alpha_{p} I$ zuordnet $\left(I=\right.$ die identische Abbildung von $\left.R_{y}\right)$. Daraus folgt, dass in den nachstehenden Formeln für ein $W_{p}$-Element auch stets eine reeilwertige $p$-lineare und alternierende Funktion $\alpha_{p}$ als Spezialfall stehen kann.

Jede Menge der Form Hom $(X, Y)$ hat eine in natürlicher Weise induzierte Struktur eines linearen Raumes. Sind $X$ und $Y$ endlichdimensional, so ist $\operatorname{dim} \operatorname{Hom}(X ; Y)=\operatorname{dim} X \operatorname{dim} Y$. Die Räume $V_{p}^{\prime}$ und $W_{p}$ sind demgemäss endlichdimensional; es ist $\operatorname{dim} V_{p}=\left(\begin{array}{c}m \\ p\end{array}\right) n$ und $\operatorname{dim} W_{P}=\left(\begin{array}{c}m \\ p\end{array}\right) n^{2}$. Als Mengen von multilinearen Funktionen ${ }^{1}$ ) können diese Räume ferner wie folgt normiert werden. Ist $f$ allgemein eine q-lineare Funktion, $f: \Pi^{q} X \rightarrow Y$, so bezeichnen wir mit $f h^{1} \ldots h^{q}$ ihren Wert für die Folge $\left(h^{1}, \ldots h^{q}\right)$ von Elementen $h^{i} \in X$. Setzt man

$$
f=\sup _{h^{i}=1} f h^{1} \ldots h^{q}
$$

so werden auf diese Weise zunächst der Raum $\operatorname{Hom}\left(R_{y} ; R_{y}\right)$ und dann die Räume (2.1) mit einer Norm versehen. Als endlichdimensionale normierte Räume sind $V_{p}^{-}$und $W_{p}$ rollständig (d.h. Banachräume).

2.2. Für $A_{p} \in W_{p}$ repräsentiert der Ausdruck

$$
L_{1}=A_{p} h^{1} \wedge \ldots \wedge h^{p}
$$

1) Es besteht ja ein kanonischer Isomorphismus von dem Raum Hom $\left(\wedge^{p} R_{x} ; Y\right)$ auf den Raum der alternierenden p-linearen Abbildungen $\pi^{p} R_{x} \rightarrow Y$; bezeichnet man die Abbildung $\left(h^{1}, \ldots, h^{p}\right) \rightarrow h^{1} \wedge \ldots \wedge h^{p}$ von $\pi^{p} R_{x}$ nach $\wedge^{p} R_{x}$ mit $\sigma$, so ist dieser Isomorphismus durch $a \rightarrow a \cdot \sigma$ für $a \in \operatorname{Hom}\left(\wedge^{p} R_{x} ; Y\right)$ gegeben. 
mit festen $h^{1}, \ldots, h^{p}$ aus $R_{x}$ eine lineare Abbildung $R_{y} \rightarrow R_{y}$; somit vertritt $L_{1} k$ für $k \in R_{y}$ einen $R_{y}$-Vektor und $L_{1} L_{2}$ für $L_{2} \in \operatorname{Hom}\left(R_{y} ; R_{y}\right)$ ein Element aus $\operatorname{Hom}\left(R_{y} ; R_{y}\right)$. Für $a_{q} \in V_{q}$ und $A_{p} \in W_{p}$ ist die durch

$$
M h^{1} \ldots h^{p+q}=A_{p} h^{1} \wedge \ldots \wedge h^{p}\left(a_{q} h^{p+1} \wedge \ldots \wedge h^{p+q}\right)
$$

definierte Funktion $M$ eine $(p+q)$-lineare Abbildung von $\Pi^{p+q} R_{x}$ in $R_{y}$. Wir definieren ein Produkt $W_{p} \times V_{q} \rightarrow V_{q+p}$ durch

$$
A_{p} \wedge a_{q}=\text { alt } M
$$

wo »alt» den alternierenden Teil der multilinearen Abbildung $M$ bezeichnet. $\left.{ }^{1}\right)$ Dasgleiche Zeichen $\wedge$ benutzen wir für das Produkt $W_{p} \times W_{q} \rightarrow W_{p+q}$, das durch die Formeln

$$
\begin{gathered}
N h^{1} \ldots h^{p+q}=A_{p}^{\prime} h^{1} \\
\wedge \ldots \wedge h^{p} \quad\left(B_{q} h^{p+1} \wedge \ldots \wedge h^{p+q}\right), \\
A_{p} \wedge B_{q}=\operatorname{alt} N
\end{gathered}
$$

für $A_{p} \in W_{p}, B_{q} \in W_{q}$ angegeben wird. Hier ist $N$ eine $(p+q)$-lineare Abbildung von $\Pi^{p+q} R_{x}$ in den Raum $\operatorname{Hom}\left(R_{y} ; R_{y}\right)$. Beide Produkte sind linear in beiden Argumenten, und es gelten die Gesetze der Assoziativität

$$
\begin{aligned}
& \left(A_{p} \wedge B_{q}\right) \wedge a_{r}=A_{p} \wedge\left(B_{q} \wedge a_{r}\right)=A_{p} \wedge B_{q} \wedge a_{r}, \\
& \left(A_{p} \wedge B_{q}\right) \wedge C_{r}=A_{p} \wedge\left(B_{q} \wedge C_{r}\right)=A_{p} \wedge B_{q} \wedge C_{r}
\end{aligned}
$$

für $a_{r} \in V_{r}, A_{p} \in W_{p}, B_{q} \in W_{q}, C_{r} \in W_{r}$.

Falls für ein Paar $A_{p} \in W_{p}, B_{q} \in W_{q}$ die $R_{y}$-Endomorphismen $A=$ $A_{p} h^{1} \wedge \ldots \wedge h^{p}$ und $B=B_{q} h^{p+1} \wedge \ldots \wedge h^{p+q}$ für jede Wahl der festen Argumenten $h^{i} \in R_{x}$ kommutieren, so dass stets $A B=B A$, so haben wir

$$
A_{p} \wedge B_{q}=(-1)^{p q} B_{q} \wedge A_{p} .
$$

Diese Formel gilt besonders im Falle, wo einer der Faktoren, etwa $A_{p}$, die Form $A_{p}=\alpha_{p} I$ hat $\left(\alpha_{p} h^{1} \ldots h^{p}\right.$ reell, $I$ ist die identische Abbildung von $R_{y}$ ). In diesem Fall kann für $B_{q}$ auch eine $R_{y}$-wertige Funktion $b_{q} \in V_{q}$ stehen.

Hinsichtlich der in den Räumen $V_{P}$ und $W_{P}$ definierten Normen haben wir die Ungleichungen durch

1) Der alternierende Teil alt $N$ einer k-linearen Funktion $N: \pi^{k} R_{x} \rightarrow Y$ wird

$$
(\operatorname{alt} N) h^{1} \ldots h^{k}=\sum_{\sigma} \frac{1}{k !}(-1)^{\sigma} N h^{i_{1}} \ldots h^{i_{k}}, h^{i} \in R_{x},
$$

definiert, wo $\sigma=\left(i_{1}, \ldots, i_{k}\right)$ eine Permutation der Folge $(1, \ldots, k)$ und $(-1)^{\sigma}=+1$ oder -1 ist, je nachdem ob die Permutation gerade oder ungerade ist. Die Summation ist über alle Permutationen zu erstrecken. 


$$
\begin{array}{ll}
\left|A_{p} \wedge a_{q}\right| \leq\left|A_{p}\right|\left|a_{q}\right| & \text { (für } A_{p} \in W_{p}, a_{q} \in V_{q} \text { ), } \\
\left|A_{p} \wedge B_{q}\right| \leq\left|A_{p}\right|\left|B_{q}\right| & \text { (für } A_{p} \in W_{p}, B_{q} \in W_{q} \text { ), }
\end{array}
$$

die leicht aus den Definitionen folgen. Aus diesen Gleichungen folgert man, dass beide Produkte in den entsprechenden Produkträumen stetig sind.

Die Produkte $A_{p} \wedge a_{q}$ und $A_{p} \wedge B_{q}$ sind für $p+q \leq m=\operatorname{dim} R_{x}$ in beiden Argumenten regulär. Unter der Regularität z.B. des Produktes $A_{p} \wedge a_{q}$ im letzten Argument versteht man, dass aus der Bedingung

$$
A_{p}^{0} \wedge a_{q}=0
$$

für ein festes $A_{p}^{0} \in W_{p}$ und für jedes $a_{q} \in V_{q}$ die Gleichung

$$
A_{p}^{0}=0
$$

folgt. Für $R_{y}=R=$ der Körper der reellen Zahlen ist die Regulärität der Produkte eine bekannte Eigenschaft, und der Beweis ist auf diesen Spezialfall etwa folgendermassen zurückführbar.

Man wähle eine Basis $e_{1}, \ldots, e_{n}$ von $R_{y}$ und nehme (2.8) an. Die Behauptung $A_{p}^{0}=0$ ist genau dann gültig, wenn für jedes $i, i=1, \ldots, n$ die $n$ Koordinaten $\alpha^{1 i} h^{1} \wedge \ldots \wedge h^{p}, \ldots, c_{p}^{n i} h^{1} \wedge \ldots \wedge h^{p}$ des $R_{y}$-Vektors $A_{p}^{0} h^{1} \wedge \ldots \wedge h^{p} e_{i}$ für $h^{1}, \ldots, h^{p} \in R_{x}$ identisch verschwinden; oder anders ausgedrückt, wenn die reellen $p$-linearen und alternierenden Funktionen $\alpha_{p}^{i j}$ verschwinden. Setzt man nun in (2.8) $a_{q}=\alpha_{q} e^{i}$ ein, wo $\alpha_{q}$ eine beliebige reellwertige alternierende Funktion bezeichnet, so erhält man die $n$ Gleichungen $\alpha_{p}^{j i} \wedge \alpha_{q}=0, j=1, \ldots, n$, und der Beweis ist somit auf den reellen Fall zurückgeführt worden.

2.3. Eine stetige $R_{y}$-wertige $p$-Form auf $U \subset R_{x}$ ist eine stetige $A b$ bildung $f_{p}: U \rightarrow V_{p}$; eine stetige Abbildung $F_{p}: U \rightarrow W_{p}$ nennen wir eine stetige $p$-Form mit Werten in Hom $\left(R_{y} ; R_{y}\right)$.

Für das Nachstehende sei $U$ eine geschlossene Kugel in $R_{x}$ mit dem Mittelpunkt $0 \in R_{x}$ und dem Radius $\varrho>0$. Den linearen Raum der stetigen $p$-Formen $f_{p}$ auf $U(p=0,1,2, \ldots)$ mit Werten in $R_{y}$ bzw. in $\operatorname{Hom}\left(R_{y} ; R_{y}\right)$ bezeichnen wir mit $\mathfrak{B}_{p}^{(s)}$ bzw. mit $\mathfrak{⿴ 囗 十}_{p}^{(s)}$. Der Raum der stetigen reellwertigen $p$-Formen $\varphi_{p}$ auf $U$ kann mit Hilfe der Einbettung $\varphi_{p}(x) \rightarrow \varphi_{p}(x) I$ für $x \in U$ als ein linearer Unterraum ron $\mathfrak{s}_{p}^{(s)}$ betrachtet werden.

Durch

$$
\| f_{p, 1}=\sup _{x \in U} f_{p}(x), \quad f_{p} \in \mathfrak{B}_{p}^{(s)}
$$

wird z.B. der Raum $\mathfrak{B}_{p}^{(s)}$ mit einer Norm versehen; entsprechend wird der Raum $\mathfrak{S}_{p}^{(s)}$ normiert. Da $U$ kompakt ist, sind beide Räume vollständig.

Mit Hilfe der in 2.2 definierten Produkte geben wir entsprechende Operationen für stetige Formen an. Für $F_{p} \in \mathfrak{S}_{p}^{(s)}, G_{q} \in \mathfrak{S}_{q}^{(s)}$ und $f_{q} \in \mathfrak{B}_{p}^{(s)}$ setzen wir 


$$
\begin{aligned}
& \left(F_{p} \wedge f_{q}\right)(x)=F_{p}(x) \wedge f_{q}(x), \\
& \left(F_{p} \wedge G_{q}\right)(x)=-F_{p}(x) \wedge G_{q}(x) .
\end{aligned}
$$

Es ist leicht zu verifizieren, dass das Resultat eine stetige Form ist, und (mit Hilfe der Ungleichungen (2.7) ) dass die Produkte stetige Funktionen sind von $\mathfrak{W}_{p}^{(s)} \times \mathfrak{B}_{q}^{(s)}$ nach $\mathfrak{S}_{p+q}^{(s)}$ und von $\mathfrak{S}_{p}^{(s)} \times \mathfrak{W}_{q}^{(s)}$ nach $\mathfrak{W}_{p+q}^{(s)}$.

Durch eine Folge $\left(x_{0}, \ldots, x_{p}\right)$ von $(p+1)$-Punkten $x_{i} \in U$ wird ein affines $p$-Simplex $s^{p}$ in $U$ angegeben. Die kleinste konvexe Menge, die die Punkte $x_{0}, \ldots, x_{p}$ enthält, nennen wir das entsprechende geometrische Simplex. Es wird auch mit $s^{p}$ bezeichnet. Der lineare Raum $\mathfrak{c}^{p}$ der $p$-Ketten $c^{p}$ von affinen Simplexen auf $U$ wird als der freie Modul über die reellen Zahlen definiert, der von der Menge $S^{p}$ der affinen $p$-Simplexe in $U$ entspannt wird. Eine $p$-Kette auf $U$ kann somit als eine »formale lineare Kombination»

$$
\sum \xi_{i} s_{i}^{p}, \quad s_{i}^{p} \in S^{p}
$$

geschrieben werden, wo nur eine endliche Anzahl der reellen Multiplikatoren $\xi_{i} \neq 0$ ist.

Für $s^{p} \subset U$ und $f_{p} \in \mathfrak{B}_{p}^{(s)}$ kann man das Integral

$$
\int_{s p} f_{p} \in R_{y}
$$

definieren (vgl. [3], III). Für eine $p$-Kette $c^{p}=\sum \xi_{i} s_{i}^{p}$ auf $U$ setzt man

$$
\int_{c p} f_{p}=\sum \xi_{i} \int_{s_{i}^{p}} f_{p} .
$$

Dieses Integral ist eine bilineare Funktion $\mathbb{C}^{p} \times \mathfrak{B}_{p}^{(s)} \rightarrow R_{y}$; da es sich um stetige $p$-Formen handelt, ist diese bilineare Funktion in der ersten Argumente regulär. $\left.{ }^{1}\right)$ Jeder Kette $c^{p} \in \mathfrak{C}^{p}$ entspricht ferner eine stetige lineare Abbildung $\quad c_{o p}^{p}: \mathfrak{B}_{p}^{(s)} \rightarrow R_{y} \quad$ mit $\quad c_{o p}^{p} f_{p}=\int_{c^{p}} f_{p} ;$ aus der Definition des Integrals und der Norm einer $p$-Form folgert man nämlich die Ungleichung $\left(s^{p} \subset U\right.$ ein $p$-Simplex $)$

$$
\int_{s^{p}} f_{p} \leq \sup _{x \in s^{p}}\left|f_{p}(x)\right| \delta^{p} \leq\left\|f_{p}\right\| \delta^{p}
$$

wo $\delta$ den Diameter des von $s^{p}$ bestimmten geometrischen Simplexes bezeichnet; hieraus folgt die Stetigkeit der linearen Abbildung $c_{o p}^{p}$.

Entsprechend wird das Integral für die Elemente von $\mathfrak{W}_{p}^{(s)}$ definiert.

1) Das heisst: $\int f_{p}=0$ für jedes $c_{p} \in \complement^{p}$ impliziert $f_{p}=0$. 
2.4. Wie üblich bezeichnen wir mit $\partial c^{p}$ den Rand einer $p$-Kette, und der Korand oder der Rotor einer $p$-Form $f_{p}$ kann folgenderweise definiert werden:

Eine stetige $(p+1)$-Form $g^{p+1}$ ist der Rotor einer stetigen $p$-Form $f_{p}$, falls die Gleichung

$$
\int_{c} g_{p+1}=\int_{\partial c p+1} f_{p}
$$

für jede $(p+1)$-Kette von affinen $(p+1)$-Simplexen auf $U$ gilt ${ }^{1}$.)

Wir bezeichnen eine solche Form $g_{p+1}$ im folgenden mit $d f_{p}$. Da das affine Integral in der Kettenargumente regulär ist, folgert man aus der obigen Definition, dass der Rotor einer stetigen Form eindeutig ist, falls er existiert.

Eine hinreichende Bedingung für die Existenz des Rotors einer $p$-Form ist, dass diese in $U$ stetig differenzierbar ist. Für eine solche Form $f_{p}$ ergibt sich

$$
\left(d f_{p}\right)(x)=\operatorname{alt} f_{p}^{\prime}(x),
$$

wo $f_{p}^{\prime}(x)$ die Ableitung der Funktion $f_{p}: U \rightarrow V_{p}$ in bezug auf $x$ bezeichnet; $f_{p}^{\prime}(x)$ ist für ein festes $x \in U$ eine $(\mathrm{p}+1)$-lineare Funktion von $\pi^{p+1} R_{x}$ in $R_{y}$.

Aus der Definition des Rotors folgt, dass $d\left(d f_{p}\right)$ existiert und gleich Null ist, falls $d f_{p}$ existiert.

Diejenigen Elemente von $\mathfrak{\Re}_{p}^{(s)}$, die einen Rotor besitzen, nennen wir reguläre $p$-Formen auf $U$. Die Menge $\mathfrak{B}_{p}^{(r)}$ aller solchen Formen ist ein linearer Unterraum von $\mathfrak{S}_{p}^{(s)}$. In der eingeführten Topologie von $\mathfrak{B}_{p}^{(s)}$ ist dieser Unterraum nicht geschlossen. Es gilt aber folgender Satz.

Es sei $\left\{f_{p}^{n}\right\}$ eine Folge von regulären p-Formen. Konvergieren die Folgen $\left\{f_{p}^{n}\right\}$ und $\left\{d f_{p}^{n}\right\}$ in $\mathfrak{B}_{p}^{(s)}$, so konvergiert $\left\{f_{p}^{n}\right\}$ gegen eine reguläre p-Form $f_{p}$ und es gilt $\left.d f_{p}=\lim _{n \rightarrow \infty} d f_{p}^{n} \cdot{ }^{2}\right)$

1) In der Tat ist es schon hinreichend, dass die Gleichung für alle $p$-Simplexe in $U$ in Kraft ist.

2) Man könnte in dem Raum $\mathfrak{2}_{p}^{(r)}$ mit gutem Cirund auch eine andere Norm benutzen: definiert man die "Sternnorm» von $f \in \mathfrak{B}_{p}^{(r)}$ durch

$$
f^{*}=\max (f, d f) \text {. }
$$

so kann man leicht verifizieren, dass es sich um eine Norm in $\mathfrak{B}_{p}^{(r)}$ handelt. Da $\|f\|^{*}<\varrho$ die Gleichung $f<\varrho$ impliziert, ist die durch die neue Norm definierte "Sterntopologie» feiner als die von uns angewandte. Insbesondere gibt es weniger Cauchy-Folgen in der Sterntopologie, und da $\left\{f_{p}^{i}\right\}$ eine Cauchy-Folge in der Sterntopologie genau dann ist, wenn die beiden Folgen $\left\{f_{p}^{i}\right\}$ und $\left\{d f_{p}^{i}\right\}$ in der Metrik von $\mathfrak{B}_{p}^{(s)}$ konvergieren, folgern wir aus dem Konvergenzsatz im Text, dass der Raum $\mathfrak{B}_{p}^{(r)}$ vollständig in der Sterntopologie ist. 
Man bezeichne mit $f_{p}$ den Grenzwert der Folge $\left\{f_{p}^{n}\right\}$ und mit $g_{p+1}$ den Grenzwert der Folge $\left\{d f_{p}^{n}\right\}$ Es sei weiter $\varepsilon>0$ gegeben und $s^{p+1} \subset U$ ein affines Simplex mit dem Diameter $\delta>0$. Wir setzen $M=\max (\delta, p+1)$. Es existieren solche natürliche Zahlen $n_{0}$ und $n_{1}$, dass

$$
\left\|f_{p}-f_{p}^{n}\right\|<\frac{\varepsilon}{2 M^{p+1}} \text { für } n>n_{0} \text { und }\left\|d f_{p}^{n}-g_{p+1}\right\|<\frac{\varepsilon}{2 M^{p+1}} \text { für } n>n_{1}
$$

ist. Für ein festes $N>\max \left(n_{0}, n_{1}\right)$ haben wir somit

$$
\begin{gathered}
\left|\int_{s^{p+1}} g_{p+1}-\int_{\partial s^{p+1}} f_{p}\right| \leq\left|\int_{p^{p+1}} g_{p+1}-\int_{s^{p+1}} d f_{p}^{N}\right|+\left|\int_{\partial s} f_{p+1}^{N}-\int_{\partial s} f_{p+1}\right| \\
\leq\left\|g_{p+1}-d f_{p}^{N}\right\| \delta^{p+1}+\| f_{p}^{N}-f_{p}(p+1) \delta^{p}<\varepsilon .
\end{gathered}
$$

Da $\varepsilon$ beliebig ist, gilt also

$$
\int_{s p-1} g_{p+1}=\int_{\partial s} f_{p}
$$

für beliebiges $s^{p+1} \subset U$, woraus das Resultat folgt.

Der Operator $d$ ist linear von $\mathfrak{B}_{p}^{(r)}$ nach $\mathfrak{B}_{p+1}^{(r)}$. Er ist nicht stetig in der induzierten Topologie von $\mathfrak{B}_{p}^{(r)}$. Eine $p$-Form $f$ mit $d f=0$ heisst geschlossen; wir bezeichnen den linearen Raum aller geschlossenen $p$ Formen - d.h. den Kern des Operators $d=d_{p}-$ mit $\mathfrak{B}_{p}^{(g)}$. Es gilt also $\mathfrak{B}_{p}^{(g)} \subset \mathfrak{B}_{p}^{(r)} \subset \mathfrak{B}_{p}^{(s)}$, und aus dem obigen Konvergenzsatz folgt, dass $\mathfrak{B}_{p}^{(g)}$ : ein geschlossener Unterraum von $\mathfrak{B}_{p}^{(s)}$ ist.

Wir haben die differentielle Folge

$$
0 \rightarrow R_{y} \stackrel{d_{-1}=j}{\longrightarrow} \mathfrak{B}_{0}^{(r)} \stackrel{d_{0}}{\longrightarrow} \ldots \stackrel{d_{m-1}}{\longrightarrow} \mathfrak{V}_{m}^{(r)} \rightarrow 0,
$$

wo also $d_{p+1} d_{p}=0$. Hier ist $j$ die kanonische Injektion, die einem Vektor von $R_{y}$ die entsprechende konstante Funktion oder 0-Form zuordnet. Gemäss dem sog. Lemma von Poincaré, das in der nächsten Nr. hergeleitet wird, i.st diese Folge sogar exakt, d.h. $\operatorname{Im} d_{p-1}=\operatorname{Ker} d_{p}=\mathfrak{B}_{p}^{(g)}$. Daher ist das Bild von $d_{p}$ ein geschlossener Unterraum in dem entsprechenden Raum $\mathfrak{S}_{p+1}^{(s)}$ der stetigen Formen.

Entsprechende Resultate sind für die Räume der reellwertigen oder $\operatorname{Hom}\left(R_{y} ; R_{y}\right)$-wertigen $p$-Formen gültig.

Bezeichnen wir den Rotor für die Elemente des Raumes $\mathfrak{W}_{p}^{(r)}$ wieder mit $d$, so haben wir die Produktregeln

$$
\begin{aligned}
& d\left(F_{p} \wedge f_{q}\right)=\left(d F_{p}\right) \wedge f_{q}+(-1)^{p} F_{p} \wedge d f_{q}, \quad F_{p} \in \mathfrak{S}_{p}^{(r)}, f_{q} \in \mathfrak{S}_{q}^{(r)}, \\
& d\left(F_{p} \wedge G_{q}\right)=\left(d F_{p}\right) \wedge G_{q}+(-1)^{p} F_{p} \wedge d G_{q}, \quad F_{p} \in \mathfrak{S}_{p}^{(r)}, G_{q} \in \mathfrak{S}_{q}^{(r)},
\end{aligned}
$$


A. I. 318

welche im Falle, dass die Formen stetig differenzierbar sind, mit Hilfe von (2.11) und der entsprechenden Formel für Elemente aus den Räumen $\mathfrak{S}_{p}^{(r)}$ durch direktes Ausrechnen bestätigt werden können. Die Formeln (2.13) enthalten insbesondere das Resultat, dass das Produkt von zwei regulären Formen wieder regulär ist.

2.5. Der Randoperator $\partial$ ist eine lineare Abbildung $\partial: \mathfrak{c}^{p} \rightarrow \mathbb{C}^{p-1}$, und wir haben die abnehmende Folge

$$
\ldots \stackrel{\partial_{1}}{\longrightarrow} \mathfrak{C}^{1} \stackrel{\partial_{0}}{\longrightarrow} \mathfrak{C}^{0} \stackrel{\pi}{\longrightarrow} R \rightarrow 0
$$

mit $\partial_{p} \partial_{p+1}=0, \pi \partial_{0}=0$; hier ist $R=R^{1}$ der eindimensionale Vektorraum der reellen Zahlen und $\pi=\partial_{-1}$ ein Epimorphismus, der zu einer 0 -Kette die Summe der Koeffizienten in ihrer Entwicklung assoziiert. Diese Folge ist bekanntlich exakt ( $U$ ist eine Kugel), d.h. Im $\partial_{p}=\operatorname{Ker} \partial_{p-1}$. Dies folgt z.B. aus den Relationen

$$
\begin{array}{ll}
c^{p} & =\partial k c^{p}+k \partial c^{p} \quad \text { für } p>0, \\
c^{0} & =\partial k c^{0}+\Sigma \cdot o ;
\end{array}
$$

hier bezeichnet $\sum=\pi\left(c^{0}\right)$ die Summe der Koeffizienten der Kette $c^{0}$ in ihrer Entwicklung und $o$ ist die von dem Nullpunkt von $R_{x}$ repräsentierte 0 -Kette. Der lineare Operator $k$ (Kegeloperator) ist durch

$$
k\left(s^{p}\right)=\left(0, x_{0}, \ldots, x_{p}\right) \quad s^{p} \subset U
$$

für affine Simplexe definiert, und durch lineare Erweiterung wird er für beliebige affine Ketten angegeben, und zwar als lineare Abbildung $k=k_{p}: \mathfrak{C}^{p} \rightarrow \mathbb{C}^{p+1}$.

Für stetige $p$-Formen hat man auch einen "Kegeloperaton", der mit $k$ in gewissem Sinn adjungiert ist. Man sucht einen Operator $q$, der jeder stetigen Form $f_{p}$ (mit $\left.p>0\right)$ eine stetige $(p-1)$-Form $q f_{p}$ zuordnet, so dass

$$
\int_{k i s p-1} f_{p}=\int_{s^{p}-1} \gamma f_{P}
$$

für jedes $s^{p-1} \subset U$ gilt. Existiert der Operator $p$, so ist er durch diese Forderung eindeutig bestimmt und linear, und man hat $\varphi_{p} \varphi_{p+1}=0$. In [3] , III. 1. 3 wird gezeigt, dass die Formel

$$
\left(\varphi f_{p}\right)(x)=\int_{0}^{1} d\left(\tau^{p}\right) f_{p}(\tau x) x
$$


den gesuchten Operator ergibt (vgl. auch [1], Exposé 7). Es können leicht die den Formeln (2.15) analogen Relationen hergeleitet werden:

$$
\begin{array}{ll}
f_{p}=d \varphi f_{p}+\varphi d f_{p} & \text { für } p>0, \\
f_{0}=j f_{0}(0)+\varphi d f_{0}, &
\end{array}
$$

wo (nach der Definition von $j) \quad j f_{0}(0)$ die konstante Funktion $b_{0}$ mit $b_{0}(x)=f_{0}(0)$ ist.

Mit den Formeln (2.19) hat man die Exaktheit der Folge (2.12). Dies enthält das Lemma von Poincaré: für eine Form $f_{p+1}$ mit $d f_{p+1}=0$ existiert stets eine $p$-Form $g_{p}$, so dass $d g_{p}=f_{p+1} \cdot{ }^{1}$ )

Man bemerke noch, dass $\varphi_{p}: \mathfrak{B}_{p}^{(r)} \rightarrow \mathfrak{B}_{p-1}^{(r)}$ stetig ist mit $\left\|\varphi_{p} f_{p}\right\| \leq p \varrho\left\|f_{p}\right\|$ $(\varrho=$ der Radius der Kugel $U$ ), welcher z.B. aus der expliziten Formel $(2.18)$ folgt. $\left.{ }^{2}\right)$

2.6. Zum Schluss dieses Paragraphen wollen wir eine obere Schranke für die Norm eines gewissen linearen Operators bestimmen. Man definiert eine lineare Abbildung $T: \mathfrak{B}_{p}^{(s)} \rightarrow \mathfrak{B}_{p}^{(s)}$ durch

$$
T f_{p}=\varphi\left(A \wedge f_{p}\right)=\int_{0}^{1} d\left(\tau^{p+1}\right)\left\{A(\tau x) \wedge f_{p}(\tau x)\right\} x,
$$

wo $A$ ein Element des Raumes $\mathfrak{S}_{1}^{(s)}$ ist und $f_{p} \in \mathfrak{B}_{p}^{(s)}$. Die $n$-te Potenz $T^{n}$ des Operators $T$ ist es für deren Norm wir eine Abschätzung haben wollen. Setzen wir

$$
m(t)=\sup _{|x| \leq t}\left|f_{p}(x)\right| \quad 0 \leq t \leq \varrho,
$$

wo also $m:[0, \varrho] \rightarrow R$ stetig und monoton ist mit $m(\varrho)=\left\|f_{p}\right\|$ (gemäss (2.9)), so erhalten wir zuerst

$$
\begin{aligned}
\left|\left(T f_{p}\right)(x)\right| & =\frac{1}{\mid x_{i}^{p}} \int_{0}^{x} d\left(t^{p+1}\right)\left\{A(t e) \wedge f_{p}(t e)\right\} e \\
& \leq \frac{\| A||}{\mid x_{i}^{p}} \int_{0}^{x} d\left(t^{p+1}\right) m(t),
\end{aligned}
$$

wo $t=|x| \tau, \quad e=|x|^{-1} x$ gesetzt worden ist.

1) Nennt man eine solche geschlossene $p$-Form total, die der Rotor einer $(p-1)$ Form ist, so kann man das obige Resultat folgenderweise aussprechen: jede auf $U$ definierte geschlossene $p$-Form ist total.

2) Es ist klar, dass man in den Definitionen von $k$ und $\varphi$ anstatt des Nullpunkts einen beliebigen inneren Punkt $t_{0}$ benutzen könnte. Die entsprechenden Operatoren werden mit $k_{t_{0}}$ und $\varphi_{t_{0}}$ bezeichnet. 
Da $\quad 0 \leq m(t) \leq\left\|f_{p}\right\|$ ist, erhält man aus Obigem insbesondere

$$
\left|\left(T f_{p}\right)(x)\right| \leq \frac{\|A\|\left\|f_{p}\right\|}{|x|^{p}} \int_{0}^{|x|} d\left(t^{p+1}\right)=|x|\|A\|\left\|f_{p}\right\| .
$$

Die Abschätzung für $\left|T^{n}\right|$ erhalten wir jetzt durch Induktion. In der Tat folgert man aus der Annahme

$$
\left|\left(T^{n-1} f_{p}\right)(x)\right| \leq \frac{p !\left\|f_{p}\right\|}{(p+n-1) !}((p+1)\|A\||x|)^{n-1},
$$

wenn man in (2.20) $T^{n-1} f_{p}$ für $f_{p}$ einsetzt

$$
\begin{aligned}
\left|\left(T^{n} f_{p}\right)(x)\right| & \leq \frac{p !\left\|f_{p}\right\|(\|A\|(p+1))^{n}}{(p+n-1) !|x|^{p}} \int_{0}^{x} t^{p+n-1} d t \\
& =\frac{p !|| f_{p} \|(\|A\|(p+1)|x|)^{n}}{(p+n) !} .
\end{aligned}
$$

Da dies für $n=1$ gilt (Gleichung $\left.(2.20)^{\prime}\right)$, gilt es für jedes $n \geq 1$. Da für $p \geq 1$ die Ungleichung $\frac{p !}{(p+n) !} \leq \frac{1}{n !}$ besteht, erhalten wir schliesslich

$$
\left\|T^{n} f_{p}\right\| \leq \frac{\left(M_{p} \varrho\right)^{n}}{n !}\left\|f_{p}\right\| \text { oder }\left\|T^{n}\right\| \leq \frac{1}{n !}\left(M_{p} \varrho\right)^{n}
$$

wo $M_{p}=\|A\|(p+1)$ gesetzt worden ist, und $\varrho$ den Radius der Kugel $U$ bezeichnet.

\section{§ 3. Fragestellung}

3.1. Es sei $A$ eine vorgegebene stetige 1-Form auf $U$ mit Werten in Hom $\left(R_{y} ; R_{y}\right)$, d.h. $A \in \mathfrak{S}_{1}^{(s)}$. Durch

$$
\psi_{p} f=d_{p} f-A \wedge f, \quad f \in \mathfrak{R}_{p}^{(r)}
$$

definiert man für $0 \leq p \leq m$ die lineare Abbildung $\psi_{p}: \mathfrak{B}_{p}^{(r)} \rightarrow \mathfrak{B}_{p+1}^{(s)}$. Wir stellen uns die Aufgabe, den Kern $\mathfrak{\Omega}_{p}$ sowie den Bildbereich $\operatorname{Im} \psi_{p}=\mathfrak{B}_{p+1}$ des Operators $\psi_{p}$ zu charakterisieren. $\mathfrak{\Omega}_{p}$ ist mit andern Worten die Menge der Lösungen $f$ der Differentialgleichung

$$
d_{p} f-A \wedge f=0, \quad f \in \mathfrak{B}_{p}^{(r)},
$$

und die Elemente von $\mathfrak{S}_{p+1}$ sind genau diejenigen regulären $(p+1)$-Formen $c$ für welche die entsprechende inhomogene Differentialgleichung 


$$
d_{p} f-A \wedge f=c
$$

in $\mathfrak{B}_{p}^{(r)}$ lösbar ist.

Ist speziell $p=0$, so ist die unbekannte Funktion $y=f$ eine stetig differenzierbare Vektorfunktion mit Werten in $R_{y}$, und (3.2) reduziert sich zu der totalen linearen Differentialgleichung

$$
y^{\prime}-A y=0
$$

Ausser der trivialen Lösung $y=0$ braucht diese Differentialgleichung bekanntlich keine Lösungen zu besitzen. Andererseits ist (3.2) für $p=m$ eine Identität, da wegen $\operatorname{dim} R_{x}=m$ jede $(p+1)$-Form in diesem Falle verschwindet.

\section{§4. Die homogene lineare Differentialgleichung}

4.1. Zunächst analysieren wir den Raum $\mathfrak{Q}_{p}$ der Lösungen der homogenen Gleichung (3.2). Aus dem Konvergenzsatz in 2.4 (und aus der Stetigkeit der Form $A$ ) folgt, das $\mathfrak{S}_{p}$ ein geschlossener linearer Unterraum von $\mathfrak{B}_{p}^{(s)}$ ist. Er ist niemals leer, da er das Nullelement des Raumes $\mathfrak{B}_{p}^{(s)}$ enthält.

Die Differentialgleichung (3.2) kann mit einem Gleichungspaar ersetzt werden, das aus einer »Integrierbarkeitsbedingung» und aus einer Integralgleichung besteht. Um dieses Gleichungspaar zu erhalten, fixieren wir eine $p$-Form $f=f_{p}$ des Lösungsraumes $\mathfrak{L}_{p}$. Wählt man einen inneren Punkt $t_{0}$ der Kugel $U$ als Anfangspunkt der Integration und "multipliziert» man die Gleichung (3.2) beiderseits mit dem Operator $d$ bzw. $\varphi_{t_{0}}$, so folgt mit Rücksicht auf die Formeln $d d=0$ und (2.19), wenn man anstatt $\varphi_{t_{0}}$ kurz $\varphi$ schreibt:

$$
d(A \wedge f)=0
$$

bzw.

$$
\begin{array}{ll}
f=d \varphi f+\varphi(A \wedge f), & p>0, \\
f=j f\left(t_{0}\right)+\varphi(A \wedge f), & p=0,
\end{array}
$$

oder, mit der Bezeichnung

$$
\begin{array}{ll}
g=d \varphi f, & p>0 \\
g=j f\left(t_{0}\right), & p=0 ; \\
f=g+\varphi(A \wedge f), & 0 \leq p \leq m .
\end{array}
$$$$
\left(\varphi=\varphi_{t_{0}}\right)
$$ 
Nach der Definition des Rotors besagt die notwendige Integrierbarkeitsbedingung (4.1), dass $\int_{\partial_{s}} A \wedge f=0$ für jedes $(p+2)$-Simplex $s^{p+2}$ in $U$ sein soll. Wir bezeichnen mit $\mathfrak{\mho}_{p}$ den linearen Raum derjenigen regulären $p$-Formen $f$, die der Gleichung (4.1) genügen. Es gilt also die Inklusion $\mathfrak{\Omega}_{p} \subset \mathfrak{\mho}_{p}$. Man sieht leicht, dass $\mathfrak{\mho}_{p}$ geschlossen in $\mathfrak{V}_{p}^{(s)}$ ist.

In der Integralgleichung (4.3) ist $g=g_{p}$ eine von der Lösung $f=f_{p}$ und von dem Anfangspunkt $t_{0}$ durch (4.2) wohlbestimmte geschlossene $p$-Form. In dem Spezialfall $p=0$ ist sie ein konstanter $R_{y}$-Vektor, nämlich der Anfangswert der Lösung $f=f_{0}=y$ der Differentialgleichung (3.4); wir nennen diese geschlossene $p$-Form $g$ im allgemeinen Fall $0 \leq p \leq m$ die zu dem Punkt $t_{0}$ gehörende Anfangsform der Lösung $f$.

Man verifiziert unmittelbar, dass umgekehrt, für eine gegebene geschlossene $p$-Form $g$, eine Lösung $f$ des Gleichungspaares (4.1), (4.3) der Differentialgleichung (3.2) genügt. Die Form $g$ ist dann die zum Punkt $t_{0}$ gehörende Anfangsform der Lösung, was aus (4.3) folgt, wenn man beachtet, dass gemäss $2.5 \varphi \varphi=0$ ist.

4.2. Wir wollen die Integralgleichung (4.3) näher besprechen, und zwar jetzt ohne die Einschränkung, dass $g$ darin geschlossen sei. Setzt man $T c=\varphi_{t_{0}}(A \wedge c)$ für $c \in \mathfrak{B}_{p}^{(s)}$ und $g=a$, so kann die Gleichung (4.3) wie folgt umgeschrieben werden

$$
(I-T) f=a
$$

Hier ist $I$ die identische Abbildung von $\mathfrak{P}_{p}^{(s)}$ auf sich selbst, und der Operator $T$ ist linear und stetig von $\mathfrak{B}_{p}^{(s)}$ in sich; nach $(2.20)^{\prime}$ haben wir $\|T\| \leq\|A\| \varrho$.

Mit Hilfe der Abschätzung (2.21) kann man in wohlbekannter Weise (vgl. z.B. [4], S. 164) zeigen, dass die Abbildung $I-T$ eine stetige Umkehrung besitzt. Mit anderen Worten heisst dies, dass die Integralgleichung (4.4) für eine gegebene stetige $p$-Form a eine eindeutige stetige $p$-Form $f$ zur Lösung hat und dass die Lösung (linear und) stetig von der Anfangsform $a$ in der Topologie von $\mathfrak{S}_{p}^{(s)}$ abhängt.

In der Tat, aus $f=a+T f$ erhält man durch $k$-malige Iteration

$$
f=\sum_{i=0}^{k} T^{i} a+T^{k+1} f .
$$

Aus der Abschätzung (2.21)

$$
\left\|T^{k}\right\| \leq \frac{M_{p}^{k} \varrho^{k}}{k !}, \quad M_{p}=(p+1)\|A\|
$$


folgt, dass die Reihe $\sum_{i=0}^{\infty} T^{i}$ in dem Banachraum der stetigen Abbildungen von $\mathfrak{B}_{p}^{(s)}$ in sich absolut konvergent ist, und bezeichnet man die Summe dieser Reihe mit $S=S_{t_{0}}$, so gilt

$$
S=(I-T)^{-1}, \quad f=S a, \quad\|S\| \leq \sum_{i=0}^{\infty}\left\|T^{i}\right\| \leq e^{\varrho M_{p}} .
$$

Mit Rücksicht auf 4.1 können wir somit folgendes über den Lösungsraum $\mathfrak{\Omega}_{p}=\operatorname{Ker} \psi_{p}$ der homogenen Differentialgleichung (3.2) aussagen. Es sei $A$ eine vorgegebene stetige 1 -Form auf $U$, mit Werten in $\operatorname{Hom}\left(R_{y} ; R_{y}\right)$, und $\Im_{p}$ der geschlossene lineare Raum derjenigen stetigen $p$-Formen $a$ (mit Werten in $R_{y}$ ), die der Bedingung $d(A \wedge a)=0$ genügen. Notwendig und hinreichend für die Existenz einer Lösung $f \in \mathfrak{B}_{p}^{(r)}$ der Differentialgleichung (3.2), die der Anfangsbedingung $d \varphi_{t_{0}} f=g$ genügt, ist dass $g \in S^{-1}\left(\widetilde{\Im}_{p}\right) \cap \mathfrak{B}_{p}^{(g)}$. Die Lösung hat den notwendigen Ausdruck $f=S g$; sie ist somit durch Angabe der Anfangsform g eindeutig bestimmt, wenn $t_{0}$ fixiert ist. Die Lösung hängt von der Anfangsform linear und stetig ab.

Die obige Bedingung für $g$ kann auch folgenderweise angegeben werden: eine geschlossene $p$-Form $g$ liefert als Anfangsform eine Lösung $f$ der Differentialgleichung (3.2) genau dann, wenn sie der Gleichung

$$
d(A \wedge S g)=0, \quad S=S_{t_{0}}
$$

genügt. Für den Raum $\Omega_{p}$ der Lösungen auf der Stufe $p$ haben wir somit den Ausdruck

$$
\mathfrak{L}_{p}=\widetilde{\mho}_{p} \cap S_{t_{\mathrm{o}}}\left(\mathfrak{B}_{p}^{(g)}\right)
$$

Der obige Satz gilt unabhängig von der Stufe $p$ der unbekannten Form $f=f_{p}$. Dagegen kann man aus dem Satz Konsequenzen ziehen, die an besondere Stufen $p$ gebunden sind; wir wollen hier zwei einfache Bemerkungen hinzufügen.

Da jede $p$-Form mit $p>m=\operatorname{dim} R_{x}$ identisch verschwindet, folgt aus (4.6):

Die Differentialgleichung (3.2) ist mit jeder geschlossenen $p$-Form $g=g_{p}$ als Anfangsform integrierbar, falls $p \geq m-1$; d.h. est ist dann $\mathfrak{\Omega}_{p}=S\left(\mathfrak{B}_{p}^{(g)}\right)$. Mit der Terminologie der Nr. 4.5 sagt man, dass die Differentialgleichung (3.2) auf den Stufen $p \geq m-1$ stets vollständig integrierbar ist.

Im Falle $p=0$, wo die geschlossenen $p$-Formen $g$ konstante $R_{y^{-}}$ Vektoren sind, folgt aus der Eindeutigkeit der (einer vorgegebenen Anfangsform entsprechenden) Lösung $f$ : Falls die Werte von zwei Lösungen $f^{1}$ und $f^{2}$ in einem Punkt von $U$ zusammenfallen, gilt es $f^{1}=f^{2}$. Dieses bekannte Sachverhältnis ist für den Fall $p=0$ charakteristisch: für $p>0$ 
können zwei Lösungen sogar in jedem Punkt einer offenen Menge zusammenfallen, ohne identisch $\mathrm{zu}$ sein. ${ }^{1}$ )

4.3. Von nun an werden wir annehmen, dass $A$ regulär ist, d.h. $A \in \mathfrak{S}_{p}^{(r)}$. Dann können wir u.a. den Lösungsraum $\mathfrak{S}_{p}$ in einer expliziteren Weise charakterisieren.

Wegen der Regularität von $A$ kann man in der Gleichung (4.1) jetzt die Produktregel (2.13) benutzen. Unter der Voraussetzung, dass $f$ eine Lösung von (3.2) ist, erhält man so zunächst die neue Integrierbarkeitsbedingung

$$
R \wedge f=0,
$$

wobei

$$
R=d A-A \wedge A
$$

gesetzt worden ist. Die 2-Form $R$ ist regulär mit Werten in Hom $\left(R_{y} ; R_{y}\right)$. Da $R$, als eine Abbildung $R \wedge$ von $\mathfrak{B}_{p}^{(s)}\left(\mathfrak{B}_{p}^{(r)}\right)$ nach $\mathfrak{B}_{p+2}^{(s)}\left(\mathfrak{B}_{p+2}^{(r)}\right)$ aufgefasst, stetig ist, ist der Kern von $R$ geschlossen in $\mathfrak{B}_{p}^{(s)}\left(\mathfrak{B}_{p}^{(r)}\right)$.

Als Lösung der entsprechenden Integralgleichung (4.3) hat $f$ ferner den notwendigen Ausdruck $f=S g$ ( $g$ geschlossen, $\left.S=S_{t_{0}}\right)$. Um umgekehrt zu zeigen, dass die Funktion $f=S g$ unter der Bedingung (4.8) tatsächlich der Differentialgleichung (3.2) genügt, bemerke man, dass der Raum $\mathfrak{S}_{p}^{(r)}$ unter $S_{t_{0}}$ stabil ist, d.h. für eine reguläre $p$-Form $a$ ist auch das Bild $S_{t_{0}} a=b$ regulär. Dies soll in der nächsten Nummer gezeigt werden. Mit Hilfe dieses Resultats erhalten wir durch Rotorbildung aus der Integralgleichung

$$
S a=a+\varphi(A \wedge S a),
$$

wo $a$ eine, zunächst beliebige $p$-Form bezeichnet und $\varphi=\varphi_{t_{0}}, S=S_{t_{0}}$ gesetzt ist,

$$
\begin{gathered}
d S a=d a+A \wedge S a-\varphi(d A \wedge S a)+\varphi(A \wedge d S a) \\
=d a+A \wedge S a-\varphi(R \wedge S a)+\varphi(A \wedge(d S a-A \wedge S a)),
\end{gathered}
$$

oder mit den in 4.2 eingeführten Bezeichnungen

$$
(I-T) \psi S a=d a-\varphi(R \wedge S a) .
$$

1) Der Spezialfall $A=O$ bietet ein unmittelbares Beispiel: alle geschlossenen Formen sind in diesem Fall Lösungen der Gleichung (3.2) und man kann leicht eine geschlossene $p$-Form konstruieren, die z.B. in einer Umgebung des Nullpunkts von $R_{x}$ gleich Null ist, ohne identisch zu verschwinden. In der Tat haben die in 4.6 konstruierten geschlossenen Formen $\Omega^{i}$ diese Eigenschaft. 
Die $(p+1)$-Form $b=\psi S a$ genügt also der Integralgleichung (4.4) wenn man dort auf der rechten Seite der Gleichung für a die Form $d a-\varphi(R \wedge S a)$ einsetzt. Wir haben somit die an und für sich wichtige allgemeine Formel

$$
\psi S a=S d a-S \varphi(R \wedge S a)
$$

Falls nun speziell $a=g$ eine geschlossene Form ist, die der Integrierbarkeitsbedingung $R \wedge S g=0$ genügt, reduziert sich die obige Gleichung zu

$$
\psi S g=0
$$

w.z.b.w.

Zusammenfassend: Während die in dem Satz von 4.2 angegebene Aussage über die Eindeutigkeit der Lösung unverändert bleibt, können wir den Existenzsatz für den Fall einer regulären Form $A$ jetzt folgenderweise umformulieren. Es sei $\mathfrak{\Re}_{p}$ der Kern der stetigen Abbildung $R \wedge$ : $\mathfrak{B}_{p}^{(r)} \rightarrow \mathfrak{B}_{p+2}^{(r)}$. Notwendig und hinreichend für die Existenz einer Lösung $f \in \mathfrak{S}_{p}^{(r)}$ der Differentialgleichung (3.2), die der Anfangsbedingung $d \varphi_{t_{0}} f=g$ genügt, ist dass $g \in S^{-1}\left(\mathfrak{I}_{p}\right) \cap \mathfrak{B}_{p}^{(g)}$.

Mit anderen Worten: für die geschlossene $p$-Form $g$ muss die Gleichung

$$
R \wedge S g=0 \quad\left(S=S_{t_{0}}\right)
$$

gelten.

Wir haben also

$$
\mathfrak{\Omega}_{p}=\mathfrak{\Re}_{p} \cap S\left(\mathfrak{B}_{p}^{(g)}\right) .
$$

Man bemerke, dass obwohl nach (4.10) und (4.7) die beiden Räume $\widetilde{\Im}_{p}$ und $\mathfrak{\pi}_{p}$ mit $S_{t_{0}}\left(\mathfrak{Q}_{p}^{(g)}\right)$ ein und denselben Raum $\mathfrak{\sim}_{p}$ als Durchschnitt haben, ist im allgemeinen $\mathfrak{\mho}_{p} \neq \mathfrak{\Re}_{p}$. In der Tat haben wir

$$
d(A \wedge f)=R \wedge f-A \wedge \psi f,
$$

so dass z.B. für $f \in \mathfrak{\Im}_{p}$ die Relation $f \in \mathfrak{\Re}_{p}$ nur in dem Falle gilt, dass $f$ der zusätzlichen Bedingung $A \wedge \psi f=0$ genügt. Immerhin hat man also $\mathfrak{\Omega}_{p} \subset \mathfrak{\Im}_{p} \cap \mathfrak{I}_{p}$.

Aus der Definition des Differentialoperators $\psi$ leitet man her (vgl. $\S 6$ ):

$$
\psi \psi a=-R \wedge a, \quad a \in \mathfrak{B}_{p}^{(r)} .
$$

Mit Rücksicht auf den obigen Existenzsatz hat man somit das Korollar: Falls $f=S g$ für $g \in \mathfrak{F}_{p}^{(g)}$ der Bedingung $\psi \psi f=0$ genügt, so ist

$$
\psi f=0 \text {. }
$$


4.4. Als Ergänzung des vorigen Abschnitts wollen wir jetzt zeigen, dass der Operator $S$ reguläre Formen zu regulären Formen transformiert, falls $A$ regulär ist. Da die inverse Abbildung $S^{-1}=I-T$ offenbar diese Eigenschaft besitzt, impliziert unsere Behauptung, dass die Restriktion von $S$ auf $\mathfrak{B}_{p}^{(r)}$ eine Bijektion von $\mathfrak{S}_{p}^{(r)}$ auf sich ist.

Wir kehren zu dem Ausdruck $S=\sum_{i=0}^{\infty} T^{i}$ zurück. Es sei $c \in \mathfrak{B}_{p}^{(r)}$. Aus der Regularität von $c$ folgt die Regularität der Formen $T^{i} c$. Um die Existenz des Rotors der Form $S c$ zu beweisen, genügt es zu zeigen, dass die Folge der Rotoren

$$
d a^{i}=\sum_{h=0}^{i} d\left(T^{h} c\right)
$$

der Formen $a^{i}=\sum_{h=0}^{i} T^{h} c$ für beliebiges $c$ aus $\mathfrak{B}_{p}^{(r)}$ in $\mathfrak{S}_{p}^{(s)}$ konvergiert (vgl. 2.4).

Aus

$$
a^{i}=\mathrm{c}+T a^{i-1}=c+\varphi\left(A \wedge a^{i-1}\right)
$$

folgt

$d a^{i}=d c+d \varphi\left(A \wedge a^{i-1}\right)=d c+A \wedge a^{i-1}-\varphi\left(d A \wedge a^{i-1}\right)+\varphi\left(A \wedge d a^{i-1}\right)$

oder

$$
d a^{i}=d c+B a^{i-1}+T d a^{i-1},
$$

wo $B: \mathfrak{B}_{p}^{(s)} \rightarrow \mathfrak{B}_{p+1}^{(s)}$ der durch $B a=A \wedge a-\varphi(d A \wedge a)$ definierte beschränkte Operator ist. Durch Wiederanwendung dieser Formel erhält man

$$
d a^{i}=\sum_{h=0}^{k} T^{h}\left(d c+B a^{i-h-1}\right)+T^{k+1} d a^{i-k-1}, 0 \leq k \leq i .
$$

Mit Hilfe dieses Ausdrucks zeigen wir zunächst, dass die Folge $\left\{d a^{i}\right\}$ beschränkt ist. Setzt man $k=i-1$ in (4.15) so wird

$$
d a^{i}=\sum_{h=0}^{i-1} T^{h}\left(d c+B a^{i-h-1}\right)+T^{i} d c
$$

Es sei $H=e^{\varrho M_{p}}$. Wegen der Abschätzungen (vgl. 2.6)

$$
\left\|T^{h}\right\| \leq \sum_{h=0}^{k}\left\|T^{h}\right\| \leq H, \quad\left\|a^{k}\right\| \leq \sum_{h=0}^{k}\left\|T^{h}\right\|\|c\| \leq H\|c\|
$$

folgern wir aus (4.16)

$$
\left\|d a^{i}\right\| \leq H(2\|d c\|+\|B\| H\|c\|),
$$

womit die Beschränktheit der Folge $\left\{d a^{i}\right\}$ bewiesen ist. 
Aus (4.15) haben wir

$$
d a^{i+j}-d a^{i}=\sum_{h=0}^{k} T^{h} B\left(a^{i+j-h-1}-a^{i-h-1}\right)+T^{k+1}\left(d a^{i+j-k-1}-d a^{i-k-1}\right)
$$

für $0 \leq k \leq i-1$. Es sei $\varepsilon>0$ gegeben. Setzt man $K=H(2\|d c\|+$ $H\|B\|\|c\|)$, so gibt es ein solches $N_{1}$ bzw. $N_{2}$ dass $\left\|a^{m}-a^{n}\right\|$ bzw. $\left\|T^{n}\right\|$ kleiner als $\varepsilon /(H\|B\|+2 K)$ für $m, n \geq N_{1}$ bzw. für $n \geq N_{2}$ ist. Es sei $N=\max \left(N_{1}, N_{2}\right)$ und $i \geq 2 N+1$. Dann haben wir nach (4.17), wo jetzt $k=N$ zu setzen ist,

$$
\begin{gathered}
\left\|d a^{i+j}-d a^{i}\right\| \leq \sum_{h=0}^{N}\left\|T^{h}\right\|\|B\|\left\|a^{i+j-h-1}-a^{i-h-1}\right\|+\left\|T^{N+1}\right\| 2 K \\
<\frac{H\|B\| \varepsilon+2 K \varepsilon}{H\|B\|+2 K}=\varepsilon
\end{gathered}
$$

was die behauptete Konvergenz ausspricht.

4.5. Es sei $A$ in (3.2) regulär und $t_{0}$ ein innerer Punkt von $U$. Wir nennen die Gleichung (3.2) auf der Stufe $p$ (mit $0 \leq p \leq m$ ) in $U$ vollständig integrierbar, wenn jede geschlossene $p$-Form $g$ die Anfangsform (in $t_{0}$ ) einer Lösung $f$ von (3.2) ist, also $\mathfrak{\Omega}_{p}=S_{t_{0}}\left(\mathfrak{S}_{p}^{(g)}\right)$. Wir werden sehen, dass dieser Begriff nicht von dem Punkt $t_{0}$ abhängt - es jst $\Omega_{p}=S_{x}\left(\mathfrak{B}_{p}^{(g)}\right)$ für jeden inneren Punkt $x$ von $U$, falls dies für einen Punkt gilt.

Ist die Differentialgleichung (3.2) auf jeder Stufe $p, 0 \leq p \leq m$, vollständig integrierbar, so sagen wir kurz, dass sie vollständig integrierbar ist.

Wir fragen nach notwendigen und hinreichenden Bedingungen, damit die Differentiagleichung (3.2) auf einer vorgegebenen Stufe $p$ vollständig integrierbar sei.

Für $p=m-1$ und $p=m$ ist dies nach 4.2 stets der Fall. Es sej nun $p<m-1$. Nach dem Resultat in 4.3 ist $R \wedge\left(\mathfrak{B}_{p}^{(r)}\right)=0$ eine hinreichende Bedingung. Sie ist offenbar mit der Voraussetzung

$$
R(x) \wedge v_{p}=0 \quad \text { jedes } x \in U, \text { jedes } v_{p} \in V_{p}
$$

äquivalent.

Wir werden sehen, dass die obige Bedingung auch notwendig ist. Es gilt ja $R \wedge f=0$ für jedes $f \in \mathfrak{\Omega}_{p}$; es ist dann offenbar, dass die vollständige Integrierbarkeit auf der Stufe $p$ die Gleichung $R(x) \wedge v_{p}=0$ für jedes $v_{p} \in V_{p}$ und für jedes $x$ in einer Umgebung von $t_{0}$ impliziert. Um dies einzusehen, nimmt man $n\left(\begin{array}{l}m \\ p\end{array}\right)$ geschlossene $p$-Formen (etwa konstante Formen) $b_{p}^{i}$, deren Werte in $t_{0}$ und daher in jedem Punkt einer Umgebung von $t_{0}$ eine Basis für $V_{p}$ bilden. Die Werte der entsprechenden 
Lösungen $f_{p}^{i}=S b_{p}^{i}$ entspannen dann in jedem Punkt einer Umgebung von $t_{0}$ den Raum $V_{p}$, da sie linear unabhängig in $t_{0}$ sind (es gilt ja $\left.f_{p}^{i}\left(t_{0}\right)=b_{p}^{i}\left(t_{0}\right)\right)$ und da die Funktionen $f_{p}^{i}: U \rightarrow V_{p}$ stetig sind. Hieraus folgt die Behauptung. Dass die Gleichung (4.18) im Falle der vollständigen Integrierbarkeit von (3.2) auf der Stufe $p$ eigentlich $\ddot{u} b e r a l l$ in $U$ in $\mathrm{Kraft}$ ist, wird in 4.6 gezeigt.

Da $t_{0}$ nicht in der Bedingung (4.18) vorkommt, folgt hieraus, dass der Begriff der vollständigen Integrierbarkeit nicht von dem Punkt $t_{0}$ abhängt.

Aus der linearen Algebra folgt nun, dass die Bedingung (4.18) sich vereinfachen lässt. Es wurde ja in 2.2 bewiesen, dass das Produkt $W_{2} \times V_{p}$ $\rightarrow V_{p+2}$ regulär für $p+2 \leq m$ ist. Ist also die Bedingung (4.18) für ein festes $p$ mit $0 \leq p \leq m-2$ in Kraft, so folgt $R(x)=0$ für jedes $x \in U$, oder

$$
R=0
$$

Zusammenfassend stellen wir fest: Die Differentialgleichung (3.2) ist auf den Stufen $p=m$ und $p=m-1$ stets vollständig integrierbar.

Falls die Differentialgleichung (3.2) auf einer Stufe $0 \leq p<m-1$ vollständig integrierbar ist, so ist sie überhaupt, d.h. auf allen Stufen, vollständig integrierbar. Eine notwendige und hinreichende Bedingung dafür ist, dass

$$
R=d A-A \wedge A=0 .
$$

Für den nichtregulären Fall wollen wir bemerken, dass die blosse Stetigkeit von $A$ mit der Annahme, dass die Gleichung (3.2) auf der Stufe $p=0$ vollständig integrierbar sei, die Regularität von $A$ impliziert. Wir wissen nicht, ob dies auch aus der Annahme der vollständigen Integrierbarkeit auf einer Stufe $p$ mit $0<p<m-1$ folgt.

4.6. Wir ergänzen den Beweis des vorigen Abschnitts indem wir zeigen: Die Bedingung

$$
R(x)=0 \quad \text { jedes } x \in U
$$

ist notwendig dafür, dass die Differentialgleichung (3.2) auf der Stufe $p$ mit $0 \leq p \leq m-2$ vollständig integrierbar sei.

Es sei $t_{0}$ ein fester innerer Punkt von $U$; man kann für das Folgende etwa $t_{0}=0$ wählen. Wir nehmen also an: es gibt für jede geschlossene $p$-Form $g$ eine Lösung der Differentialgleichung (3.2), so dass $g$ die Anfangsform von $f$ im Punkte 0 ist. Die Behauptung (4.18)' ist für $p:=0$ eine direkte Folgerung der Einzigkeit der Lösung; wir können uns somit auf den Fall $0<p<m-1$ beschränken. 
Es ist zuerst klar, dass $R(0)=0$ ist. Es sei dann $x_{0}$ ein innerer Punkt von $U, x_{0} \neq 0$. Wir wollen zeigen, dass unter unserer Annahme $n\left(\begin{array}{c}m \\ p\end{array}\right)$ Lösungen von (3.2) existieren, deren Werte in jedem Punkt eines offenen Intervalls $] x_{0},(1+\Delta) x_{0}$ [ mit $\Delta>0$ eine Basis des Raumes $V_{p}$ bilden. Für Punkte dieses Intervalls hat man folglich (wegen (4.8) und der Regularität des in Frage stehenden Produktes) $R(x)=0$, und da $R$ stetig ist, erhält man daraus $R\left(x_{0}\right)=0$. Da $x_{0}$ ein beliebiger innerer Punkt von $U$ war, ist also $R(x)=0$ für jedes $x \in U$.

Ohne Einschränkung der Allgemeinheit können wir den Raum $R_{x}$ als euklidisch annehmen; man bezeichne mit $(h, k)$ das innere Produkt zweier $R_{x}$-Vektoren $h$ und $k$. Es sei $e=\left|x_{0}\right|^{-1} x_{0}$. Wir wählen eine orthonormierte Basis $a^{1}, \ldots, a^{m}$ von $R_{x}$, die symmetrisch in bezug auf den Vektor $e$ belegen ist, d.h. so dass

$$
e=\frac{1}{\sqrt{m}} \sum_{i=1}^{m} a^{i}
$$

Es gilt also $\left(e, a^{i}\right)=\frac{1}{\sqrt{m}}, i=1, \ldots, m$.

Durch die Gleichungen

$$
\begin{array}{ll}
\Omega^{i}(x) h=\sqrt{m}\left(x-x_{0}, a^{i}\right)\left(h, a^{i}\right) & \text { für }\left(x-x_{0}, a^{i}\right)>0, \\
\Omega^{i}(x) h=0 & \text { für }\left(x-x_{0}, a^{i}\right) \leq 0
\end{array}
$$

definieren wir die $m$ reellwertigen, stetig differenzierbaren 1-Formen $\Omega^{1}, \ldots, \Omega^{m} ;$ als Ableitungen der durch

$$
\begin{array}{ll}
c^{i}(x)=\frac{\sqrt{m}}{2}\left(x-x_{0}, a^{i}\right)^{2} & \text { für }\left(x-x_{0}, a^{i}\right)>0, \\
c^{i}(x)=0 & \text { für }\left(x-x_{0}, a^{i}\right) \leq 0,
\end{array}
$$

definierten reellen Funktionen $c^{1}, \ldots, c^{m}$ sind diese Formen geschlossen. Besonders gilt auf der Strecke $x=x_{0}+\lambda e$

$$
\begin{array}{ll}
\Omega^{i}\left(x_{0}+\lambda e\right) h=\lambda\left(h, a^{i}\right) & \text { für } \lambda>0, \\
\Omega^{i}\left(x_{0}+\lambda e\right) h=0 & \text { für } \lambda \leq 0 .
\end{array}
$$

Für jedes feste $\lambda>0$ bilden die Elemente $\Omega^{1}\left(x_{0}+\lambda e\right), \ldots, \Omega^{m}\left(x_{0}+\lambda e\right)$ eine Basis des zu $R_{x}$ dualen Raumes $\operatorname{Hom}\left(R_{x} ; R\right)$.

Es sei $e^{1}, \ldots, e^{n}$ eine Basis für den Raum $R_{y}$. Für ein festes $p$ mit $0<p<m-1$ betrachten wir die $N=n\left(\begin{array}{l}m \\ p\end{array}\right) p$-Formen

$$
g_{i_{1}}^{j} \ldots i_{p}=e^{j} \Omega^{i_{1}} \wedge \ldots \wedge \Omega^{i_{p}}
$$


mit $1 \leq i_{1}<i_{2} \ldots<i_{p} \leq m, j=1, \ldots, n$. Für jedes feste $\lambda>0$ stellen die Elemente $g_{i_{1}}^{j} \ldots i_{p}\left(x_{0}+\lambda e\right)$ eine Basis für $V_{p}$ dar. Für $\lambda \leq 0$ ist $g_{i_{1}}^{j} \ldots i_{p}\left(x_{0}+\lambda e\right)=0$. Da ferner die 1-Formen $\Omega^{1}, \ldots, \Omega^{m}$ geschlossen sind, folgt aus der Produktregel (2.13) dass die $p$-Formen $g_{i_{1} \ldots i_{p}}^{j}$ auch geschlossen sind.

Durch

$$
M_{i_{1} \ldots i_{p}} h^{1} \ldots h^{p}=\left(h^{1}, a^{i_{1}}\right) \ldots\left(h^{p}, a^{i_{p}}\right)
$$

definiert man eine multilineare Funktion $M_{i_{1} \ldots i_{p}}: \pi^{p} R_{x} \rightarrow R$. Es gilt dann für $\lambda \geq 0$

$$
g_{i_{1} \ldots i_{p}}^{j}\left(x_{0}+\lambda e\right) h^{1} \wedge \ldots \wedge h^{p}=e^{j} \lambda^{p}\left(\text { alt } M_{i_{1} \ldots i_{p}}\right) h^{1} \wedge \ldots \wedge h^{p} .
$$

Aus der Definition von $M_{i_{1} \ldots i_{p}}$ folgt, dass der Wert des Ausdrucks alt $M_{i_{1} \ldots i_{p}} h^{1} \wedge \ldots \wedge h^{p} \quad$ unverändert bleibt, wenn man in ihm die Vektoren $h^{i}$ durch ihre orthogonalen Projektionen in dem von den Vektoren $a^{i_{1}}, \ldots, a^{i_{p}}$ erzeugten Unterraum $R_{x}^{i_{1} \ldots i_{p}}$ ersetzt. Daher haben wir

$$
\begin{aligned}
& v_{i_{1} \ldots i_{p}}=\max _{\left|h^{i}\right|=1, h^{i} \in R_{x}} \quad \mid\left(\text { alt } M_{i_{1} \ldots i_{p}}\right) h^{1} \wedge \ldots \wedge h^{p} \mid
\end{aligned}
$$

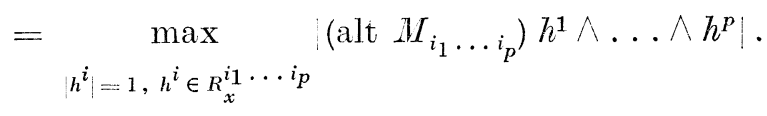

Der letzte Ausdruck hängt offenbar nur von der Dimension $p$ ab; daher ist $v_{i_{1} \ldots i_{p}}=v_{p}>0 .{ }^{1}$ )

Für die Norm der Werte der Funktionen $g_{i_{1}}^{j} \ldots i_{p}$ auf der Strecke $x=x_{0}+\lambda e$ haben wir jetzt (falls die verschiedenen Folgen $\left(j, i_{1}, \ldots, i_{p}\right)$ mit (1) $, \ldots,(N)$ bezeichnet werden und $\left|e^{j}\right|=1$ gewählt wird

$$
\begin{aligned}
\left|g^{(k)}\left(x_{0}+\lambda e\right)\right| & =\sup _{\left|h^{i}\right|=1}\left|g^{(k)}\left(x_{0}+\lambda e\right) h^{1} \wedge \ldots \wedge h^{p}\right| \\
& =\left|e^{j}\right| \lambda^{p} v_{p}=\lambda^{p} v_{p} .
\end{aligned}
$$

Falls nun unsere Differentialgleichung (3.2) auf der Stufe $p$ im Punkte $O \in R_{x}$ vollständig integrierbar ist, entsprechen den oben konstruierten geschlossenen $p$-Formen $g^{(1)}, \ldots, g^{(-)}$wohlbestimmte Lösungen $f^{(1)}, \ldots, f^{(n)}$ der Differentialgleichung, die als Lösungen der entsprechenden Integralgleichung (4.3) erhalten werden. Wir hehaupten, dass es ein $\varepsilon>0$ gibt mit der Eigenschaft, dass die $V_{p}$-Vektoren $f^{(1)}(x), \ldots, f^{(N)}(x)$ für jedes feste $x$ der Strecke $l_{\varepsilon}:\left(x=x_{0}+\lambda e, 0<\lambda \leq \varepsilon\right)$ linear unabhängig sind.

1) Die Zahl $v_{p}$ kann als das maximale Volumen eines $p$-Simplexes $s^{p}=\left(0, h^{1}, \ldots, h^{p}\right)$ in $R_{x}$ mit $\left|h^{i}\right|=1, i=1, \ldots, p$, gedeutet werden (vgl. [3], S. 67). 
Nach (4.3) haben wir

$$
f^{(i)}\left(x_{0}+\lambda e\right)=g^{(i)}\left(x_{0}+\lambda e\right)+\varphi_{0}\left(A \wedge f^{(i)}\right)\left(x_{0}+\lambda e\right) .
$$

Setzt man

$$
\beta^{i}(\lambda)=\max _{0 \leqq \tau \leqq \lambda}\left|f^{(i)}\left(x_{0}+\tau e\right)\right|
$$

und berücksichtigt man, dass $g^{(i)}(x)$ und folglich auch $f^{(i)}(x)$ auf der Strecke $\left(x=x_{0}+\lambda e, \quad-\left|x_{0}\right| \leq \lambda \leq 0\right)$ verschwindet, so erhält man $(\operatorname{vgl} .(2.20))$

$$
\begin{aligned}
\left|\varphi\left(A \wedge f^{(i)}\right)\left(x_{0}+\lambda e\right)\right| & =\frac{p+1}{\left(\left|x_{0}\right|+\lambda\right)^{p}}\left|\int_{0}^{\left|x_{0}\right|+i} t^{p}\left\{A(t e) \wedge f^{(i)}(t e)\right\} e d t\right| \\
& =\frac{p+1}{\left(\left|x_{0}\right|+\lambda\right)^{p}}\left|\int_{\left|x_{0}\right|}^{\left|x_{0}\right|+\lambda} t^{p}\left\{A(t e) \wedge f^{(i)}(t e)\right\} e d t\right| \\
& \leq(p+1)\|A\| \beta^{i}(\lambda) \lambda
\end{aligned}
$$

also

$$
\left|f^{(i)}\left(x_{0}+\lambda e\right)-g^{(i)}\left(x_{0}+\lambda e\right)\right| \leq(p+1)\|A\| \beta^{i}(\lambda) \lambda
$$

und

$$
\left|f^{(i)}\left(x_{0}+\lambda e\right)\right| \leq\left|g^{(i)}\left(x_{0}+\lambda e\right)\right|+(p+1)\|A\| \beta^{i}(\lambda) \lambda .
$$

Gemäss (4.19) ist $\left|g^{(i)}\left(x_{0}+\lambda e\right)\right|$, folglich auch die rechte Seite der letzten Ungleichung eine wachsende Funktion von $\lambda$. Daher ist

$$
\beta^{i}(\lambda) \leq\left|g^{(i)}\left(x_{0}+\lambda e\right)\right|+(p+1)\|A\| \beta^{i}(\lambda) \lambda,
$$

oder, für $0<\lambda<1 /(2(p+1) \| A)$,

$$
\beta^{i}(\lambda) \leq \frac{g^{(i)}\left(x_{0}+\lambda e\right)}{1-(p+1) A_{\lambda}}<2 g^{(i)}\left(x_{0}+\lambda e\right)
$$

Mit Rücksicht auf (4.20) haben wir somit

$$
\begin{aligned}
\left|f^{(i)}\left(x_{0}+\lambda e\right)-g^{(i)}\left(x_{0}+\lambda e\right)\right| & \leq 2(p+1)\|A\|\left|g^{(i)}\left(x_{0}+\lambda e\right)\right| \lambda \\
& =2(p+1)\|A\| \lambda^{p+1} v_{p} .
\end{aligned}
$$

Es sei jetzt $D: \pi^{N} V_{p} \rightarrow R$ eine $N$-lineare nicht ausgeartete alternierende Abbildung. Für $v^{i} \in V_{p}, i=1, \ldots, N$, ist

$$
D v^{1} \ldots v^{N}=0
$$

dann und nur dann, wenn $\left(v^{1}, \ldots, v^{N}\right)$ eine linear unabhängige Menge von Vektoren in $V_{p}$ ist. Besonders ist also 


$$
D g^{(1)}\left(x_{0}+\lambda e\right) \ldots g^{(N)}\left(x_{0}+\lambda e\right) \neq 0 \text { für } \lambda>0 .
$$

Nach (4.19) gilt

$$
D g\left({ }^{1}\right)\left(x_{0}+\lambda e\right) \ldots g^{(N)}\left(x_{0}+\lambda e\right)=\lambda^{N_{p}} D g^{(1)}\left(x_{0}+e\right) \ldots g^{(N)}\left(x_{0}+e\right) \text {. }
$$

Da $D$ stetig von $\pi^{N} V_{p}$ nach $R$ ist, existiert ein solches $\delta>0$, dass die Menge $\left(v^{1}, \ldots, v^{N}\right)$ von Vektoren aus $V_{p}$ noch linear unabhängig ist, wenn die Gleichungen

$$
\left|v^{i}-g^{(i)}\left(x_{0}+e\right)\right|<\delta, \quad i=1, \ldots, N,
$$

in Kraft sind.

Da nach (4.19) $g^{(i)}\left(x_{0}+\lambda e\right)=\lambda^{p} g^{(i)}\left(x_{0}+e\right)$ ist, folgert man aus den Ungleichungen

$$
\left|w^{i}-g^{(i)}\left(x_{0}+\lambda e\right)\right|<\lambda^{p} \delta, \quad i=1, \ldots, N \text { für } \lambda>0,
$$

dass die Menge $w^{1}, \ldots, w^{N}$ von $V_{p}$-Vektoren linear nnabhängig ist; für die Vektoren $v^{i}=\frac{1}{\lambda^{p}} w^{i} \quad(i=1, \ldots, N)$ ist nämlich dann (4.22) gültig und folglich

$$
D w^{1} \ldots w^{N}=\lambda^{p N} D v^{1} \ldots v^{N} \neq 0 .
$$

Aus (4.21) folgt nun schliesslich, dass (4.23) für die Werte $w^{i}$ $=f^{(i)}\left(x_{0}+\lambda e\right), i=1, \ldots, N$, in Kraft ist und $f^{(1)}\left(x_{0}+\lambda e\right), \ldots$, $f^{(N)}\left(x_{0}+\lambda e\right)$ also linear unabhängig sind, falls gleichzeitig

$$
2(p+1)\|A\| \lambda^{p+1} v_{p}<\lambda^{p} \delta \text { und } 0<\lambda<1 /(2(p+1)\|A\|) \text {, }
$$

d.h. falls der Punkt $x_{0}$ sich auf der Strecke $l_{\varepsilon}:\left(x=x_{0}+\lambda e, 0<\lambda<\varepsilon\right)$ mit

$$
\varepsilon=\min \left(\frac{1}{2(p+1)\|A\|}, \frac{\delta}{2(p+1)\|A\| v_{p}}\right)
$$

befindet. Damit ist der Beweis zu Ende.

\section{§ 5. Die allgemeine lineare Differentialgleichung}

Wir wollen jetzt den zweiten Teil der in $\S 3$ aufgestellten Aufgabe in Angriff nehmen. Es handelt sich um die Charakterisierung des Bildes $\mathfrak{B}_{p+1}=\operatorname{Im} \psi_{p}$. Um die Betrachtungen einfacher zu machen, beschränken wir uns auf den Fall, dass $A$ regulär ist. Man kann das Problem auch folgenderweise formulieren: Für welche regulären $(p+1)$-Formen $a$ besitzt die inhomogene Differentialgleichung 


$$
\psi f=d f-A \wedge f=a, \quad \psi=\psi_{p}
$$

Lösungen $f$ in $\mathfrak{B}_{p}^{(r)}$ ? Es ist klar, dass falls für die gegebene $(p+1)$-Form a eine Lösung $f$ existiert, die Menge $f+\mathfrak{\Omega}_{p}$ dann die Gesamtheit der Lösungen repräsentiert.

Man kann das Problem dem homogenen Fall entsprechend behandeln. Man wähle einen inneren Punkt $t_{0}$ von $U$ als Anfangspunkt der Integration und setze $\varphi_{t_{0}}=\varphi, \quad S_{t_{0}}=S$. Die Differentialgleichung (5.1) kann mit dem Gleichungspaar

$$
\begin{gathered}
-R \wedge f=\psi a \\
(I-T) f=g+\varphi a \quad(T f=\varphi(A \wedge f))
\end{gathered}
$$

ersetzt werden, wo $g$ die durch

$$
\begin{array}{ll}
g=d \varphi f & \text { für } p>0, \\
g=j f\left(t_{0}\right) & \text { für } p=0
\end{array}
$$

definierte Anfangsform der Lösung $f$ bezeichnet.

In der Tat wird, falls $f=f_{p}$ für $a=a_{p+1}$ eine vorgegebene Lösung von (5.1) ist, die Gleichung (5.2) bzw. (5.3) aus (5.1) durch "Multiplikation» mit dem Operator $d$ bzw. $\varphi$ gewonnen. Man nehme dann an, um die Implikation in der anderen Richtung zu beweisen, dass zwei reguläre Formen $f=f_{p}$ und $a=a_{p+1}$ für eine geschlossene Form $g=g_{p}$ dem Gleichungspaar $(5.2-3)$ genügen. Wegen $S=(I-T)^{-1}$ erhält man aus (5.3)

$$
f=S(g+\varphi a)
$$

und mit Hilfe der Formel (4.9) folgert man aus dieser Gleichung und aus (5.2), dass $\psi f=a$. Wenn man noch bemerkt, dass aus (5.3) die Gleichungen (5.4) für die geschlossene $p$-Form $g$ folgen (die also besagen, dass $g$ die Anfangsform in $t_{0}$ für $f$ ist), ist der Beweis zu Ende.

Substituiert man die Gleichung $(5.3)^{\prime}$ in (5.2), so wird

$$
-R \wedge S(g+\varphi a)=\psi a
$$

oder

$$
(\psi+R \wedge S \varphi) a=-R \wedge S g .
$$

Für $a \in \operatorname{Im} \psi_{p}=\mathfrak{B}_{p+1}$ gilt also

$$
(\psi+R \wedge S \varphi) a \in R \wedge S\left(\mathfrak{B}_{p}^{(g)}\right) .
$$


Diese Bedingung ist auch hinreichend dafür, dass die $(p+1)$-Form $a$ dem Bildbereich $\mathfrak{B}_{p+1}$ von $\psi_{p}$ angehört. Ist nämlich

$$
(\psi+R \wedge S \varphi) a=-R \wedge S g \quad \text { mit } g \in \mathfrak{B}_{p}^{(g)},
$$

so hat man für $f=S(g+\varphi a)$ die Gleichungen (5.2-3).

Daher ist $\psi f=a$, w.z.b.w.

Das Kriterium (5.6), obwohl es kompliziert ist, scheint ungefähr das beste zu sein, das unter unseren allgemeinen Annahmen angegeben werden kann. Falls besonders $R=O$ ist, reduziert sich die Bedingung zu

$$
\psi a=0, \quad a \in \mathfrak{B}_{p+1}^{(r)},
$$

und man hat so das Analogon des sog. Lemmas von Poincaré (vgl. 2.5).

\section{§ 6. Zwei differentielle Folgen}

In diesem Paragraphen sollen zwei differentielle Folgen mit dem Operator $\psi$ (oder eigentlich mit der 1-Form $A$ ) assoziiert werden.

Aus der Definition $\psi_{p} f=d f-A \wedge f\left(f \in \mathfrak{S}_{p}^{(r)}\right)$ folgt nach der Regel $(2.13)$

$$
d \psi_{p} f=-d A \wedge f+A \wedge d f=-R \wedge f-A \wedge \psi_{p} f
$$

oder

$$
\psi_{p+1} \psi_{p} f=-R \wedge f \quad f \in \mathfrak{B}_{P}^{(r)} .
$$

Sei jetzt $f \in \mathfrak{\Re}_{p}$, wo $\mathfrak{\Re}_{p}$ den Kern der Abbildung $R \wedge: \mathfrak{B}_{p}^{(r)} \rightarrow \mathfrak{Q}_{p+2}^{(r)}$ bezeichnet. Da gemäss obigem $\psi_{p+1} \psi_{p} f=0$ ist, gehört $\psi_{p} f \operatorname{dem}$ Kern $\Omega_{p+1}$ von $\psi_{p+1}$ an. Da nun $\Omega_{p+1} \subset \Re_{p-1}$, definiert $\psi_{p}$ also eine Abbildung von $\mathfrak{I}_{p}$ nach $\mathfrak{K}_{p+1}$, die wir wieder mit $\psi_{p}$ bezeichnen. Nach (6.1) ist die entsprechende Folge

$$
0 \rightarrow \operatorname{Ker} \psi_{0} \stackrel{\psi_{-1}=j}{\longrightarrow} \mathfrak{\Re}_{0} \stackrel{\psi_{0}}{\longrightarrow} \mathfrak{I}_{1} \stackrel{\psi_{1}}{\longrightarrow} \ldots \stackrel{\psi_{m-1}}{\longrightarrow} \mathfrak{\Re}_{m} \rightarrow 0
$$

differentiell, d.h. $\psi_{p+1} \psi_{p}=0$. Es ist hier $\mathfrak{R}_{m-1}=\mathfrak{B}_{m-1}^{(r)}$ und $\mathfrak{\Re}_{m}=\mathfrak{S}_{m}^{(r)}$.

Wir wollen jetzt die Einschränkung von $d_{p}$ auf den Raum $S^{-1}\left(\Re_{p}\right)$ $\left(S=S_{t_{0}}\right)$ untersuchen. Nach (4.9) haben wir für $f \in \mathfrak{B}_{p}^{(r)}$

$$
\psi_{p} S f=S d_{p} f-S \varphi(R \wedge S f)
$$

so dass

$$
\psi_{p} S f=S d_{p} f \quad \text { für } f \in S^{-1}\left(\Re_{p}\right)
$$


gilt. Da also $d_{p} f=S^{-1}\left(\psi_{p} S f\right)$ und $\psi_{p} S f \in \Re_{\mathrm{p}+1}$ für $f \in S^{-1}\left(\Re_{p}\right)$ gilt, ist die Restriktion von $d_{p}$ auf $S^{-1}\left(\Re_{p}\right)$ eine Abbildung nach $S^{-1}\left(\Re_{\mathrm{p}+1}\right)$, die wir wieder mit $d_{p}$ bezeichnen. Die entsprechende Folge

$$
0 \rightarrow S^{-1}\left(\operatorname{Ker} \psi_{0}\right) \stackrel{d_{-1}=j}{\longrightarrow} S^{-1}\left(\Re_{0}\right) \stackrel{d_{0}}{\longrightarrow} \ldots \stackrel{d_{m-1}}{\longrightarrow} S^{-1}\left(\Re_{m}\right) \rightarrow 0
$$

ist wegen $d_{p+1} d_{p}=0$ differentiell. Die Formel (6.3) besagt (da $S$ schon linear und bijektiv ist), dass $S$ einen Komplex-Isomorphismus von $S^{-1}(\mathfrak{K})$ auf $\mathfrak{\AA}$ definiert, wo $\Re=\sum_{i=1}^{m} \Re_{i}$ ist, mit $\AA_{-1}=\operatorname{Ker} \psi_{0}$.

Ist speziell $R=O$ (d.h. die Gleichung (3.2) vollständig integrierbar), so gilt $S^{-1}\left(\mathfrak{R}_{p}\right)=\mathfrak{\Re}_{p}=\mathfrak{V}_{p}^{(r)}$ für $p \geq 0$ und die Folge (6.4) fällt mit der exakten Folge (2.12) zusammen. Daher ist auch die isomorphe Folge (6.2) in diesem Fall exakt - ein Resultat, das wir schon am Ende des vorigen Paragraphen erhielten.

Im allgemeinen wird die Folge (6.2) nicht exakt sein, also können die entsprechenden Kohomologieräume $H^{p}(A, U)=\operatorname{Ker} \psi_{p} / \operatorname{Im} \psi_{p-1} \quad$ von Null verschieden sein.

Neben der Abbildung $\psi_{p}: \mathfrak{B}_{p}^{(r)} \rightarrow \mathfrak{B}_{p+1}^{(r)}$ kann man den durch die Gleichung

$$
\psi_{p}^{L} F=d F-A \wedge F, \quad F \in \mathfrak{W}_{p}^{(r)}
$$

definierten linearen Operator $\psi_{p}^{L}: \mathfrak{S}_{p}^{(r)} \rightarrow \mathfrak{W}_{p+1}^{(r)}$ betrachten. Eine der oben durchgeführten vollends analoge Diskussion kann erfolgen; ohne abermals alle Resultate herzuleiten bemerken wir, dass man insbesondere die der Folge (6.2) analoge differentielle Folge

$$
0 \rightarrow \operatorname{Ker} \psi_{0}^{L} \stackrel{\psi_{-1}^{L}=j^{L}}{\longrightarrow} \AA_{0}^{L} \stackrel{\psi_{0}^{L}}{\longrightarrow} \ldots \stackrel{\psi_{m-1}^{L}}{\longrightarrow} \Re_{m}^{L} \rightarrow 0
$$

erhält, wo $\Re_{p}^{L}$ den Kern des Operators $R:: \mathfrak{i s}_{p}^{(r)} \rightarrow \mathfrak{N}_{p \rightarrow 2}^{(r)}$ bezeichnet. Es gilt der folgende Satz: Der Komplex $\hat{\Omega}^{L}=\sum_{i=1}^{m} \hat{\Omega}_{i}^{L}$ ist dem Komplex $\pi^{n} \Re=\sum_{i=-1}^{m} \pi^{n} \Re_{i}$ isomorph.

Zum Beweis nehme man eine Basis $\left(e^{1}, \ldots, e^{n}\right)$ des Raumes $l_{y}$. Jedes $e^{i}$ definiert eine lineare Abbildung $\varkappa_{i}: \Re_{p}^{L} \rightarrow \AA_{p}$ durch

$$
\varkappa_{i}(F)=F e^{i} \in \Re_{p}, \quad F \in \Re_{p}^{L} .
$$

Der gesuchte Isomorphismus wird als Produkt $\tau \varkappa_{i}$ definiert. Es wird dem Leser überlassen, zu verifizieren, dass ein Komplex-Isomorphismus besteht. Insbesondere haben wir für die entsprechenden Kohomologieräume

$$
H_{L}^{p}(A, U)=\pi^{n} H^{p}(A, U) .
$$


Es ist klar, dass die obigen Betrachtungen für eine beliebige konvexe kompakte Punktmenge $V \subset U$ wesentlich durchführbar sind. Ohne hier auf Einzelheiten einzugehen, bemerken wir, dass man aus den Komplexen $\mathfrak{N}(V)$ mit $t_{0} \in V$ durch einen geejgneten Limesprozess einen Komplex $\mathfrak{n}\left(t_{0}\right)$ erhält, indem man den Bereich $V$ zu dem inneren Punkt $t_{0}$ von $U$ zusammenschrumpfen lässt. Die Kohomologie des Komplexes $\mathfrak{\Re}\left(t_{0}\right)$ ist dann eine partielle lokale Charakterisierung der 1-Form $A$ im Punkt $t_{0}$; diese Kohomologie ist insbesondere dann trivial, wenn die Gleichung (3.2) in irgendeiner Umgebung von $t_{0}$ vollständig integrierbar ist.

\section{Literatur}

[1] Cartax, H.: Séminaire de topologie algebrique 1948/49, E.N.S., 2e édition 1955.

[2] Grafub, W.: Lineare Algebra, Grundlehren Math. Wiss. XCVII, Springer, 1958.

[3] Nevandinna, F. und R.: Absolute Analysis, Grundlehren Math. Wiss. 102, Springer, 1959.

[4] Taylor, A.: Introduction to Functional Analysis, New York. John Wiley \& Sons, Ine., 1959. 